\title{
SEARCHES FOR SIGNATURES FROM GAUGE MEDIATED SUSY BREAKING
}

\author{
NAOKO KANAYA \\ University of Victoria, Physics and Astronomy \\ PO Box 3040 STN CSC Victoria BC V8W 3N\%, CANADA \\ E-mail: nkanaya@uvic.ca
}

Gauge-mediated SUSY breaking is an attractive model both theoretically and experimentally. In this paper, gauge-mediated SUSY breaking signatures studied with the OPAL experiment are summarized.

\section{Introduction}

The Standard Model (SM) of particle physics describes presently known phenomena successfully, and no distinct deviations from the SM have been confirmed. We, however, expect the SM will be extended to explain phenomena such as the electroweak symmetry breaking, baryon asymmetry and missing mass of the universe. The successful supersymmetry (SUSY) not only solves the above problems, but also provide consistent predictions with the existing experimental results. It's essential to observe a SUSY particle expected in the theories to verify the supersymmetry.

In the gauge-mediated SUSY breaking (GMSB) model ??, our world consists of three sectors, hidden sector responsible for SUSY breaking and visible sector composed of the SM particle and their super partners, and the messenger sector, which is responsible for the communication of the SUSY breaking effects into the visible sector. The SUSY particle mass spectrum is controlled by known gauge interactions. Consequently the first and second generation particles are degenerate in their masses, and dangerous flavor changing neutral currents can be avoided.

In the minimal case, the low-energy phenomenology can be described by six parameters,

$$
\Lambda, \quad M, \quad n, \sqrt{F}, \tan \beta, \operatorname{sign}(\mu) .
$$

The effective SUSY breaking scale, $\Lambda$, determines the whole SUSY particle mass scale. The SUSY particle masses depend on the messenger scale $M$ only logarithmically, and sfermions (gauginos) become light as $M$ is small (large). The parameter $\tan \beta$ affects the mixing between left- and right- handed 
sfermions, and the case $\mu<0$ gives a larger mixing than the case $\mu>0$ in the notation of positive trilinear terms.

One of the most important features of GMSB models is the fact that the gravitino is very light and becomes the lightest supersymmetric particle (LSP) while its coupling to the SUSY particle is enhanced. This is because the SUSY breaking scale $F$ is low, comparing to that of the gravity-mediated models, to give $m_{\text {soft }}$ the right order of magnitude. The gravitino mass is given by

$$
m_{\tilde{G}}=\frac{F}{\sqrt{3} M_{P}}=\left(\frac{\sqrt{F}}{100 \mathrm{TeV}}\right)^{2} \times 2.4 \quad \mathrm{eV},
$$

where $M_{P}=2.4 \times 10^{18} \mathrm{GeV}$ is reduced Planck mass. The decay length of the next-to-LSP (NLSP) depends on the gravitino mass (SUSY breaking scale),

$$
L=1.76 \times 10^{-3} \times\left(\frac{m_{\tilde{G}}}{1 \mathrm{eV}}\right)^{2}\left(\frac{m_{\mathrm{NLSP}}}{100 \mathrm{GeV}}\right)^{-5}(\beta \gamma)_{\mathrm{NLSP}} \quad \mathrm{cm} .
$$

The $\kappa_{\gamma}$ is the bino component of neutralino and is unity for sleptons. Expected experimental topologies are changed by the gravitino mass. The NLSP might decay very close to the $e^{+} e^{-}$interaction point ( "short lifetime") or in tracking detector volumes ( "intermediate lifetime") or outside of the detector ("long lifetime").

GMSB models provide some different collider signatures from those of the gravity-mediated models. Various analyses sensitive to the GMSB signatures have been performed by the LEP and Tevatron experiments??, but no indication has been observed in spite of their efforts. In this paper, the GMSB topologies studied at the OPAL experiment are introduced briefly. Some details of analysis techniques and results have been published on Refs????.

\section{GMSB signatures at $e^{+} e^{-}$collider}

The GMSB models have a very predictive mass spectrum, and the NLSP is the lightest neutralino or sleptons in the most of parameter space. The masses of the first and second generations are degenerate while the third generations can be lighter than the others due to their large Yukawa couplings. Thus the lightest stau can solely become the NLSP if $\tan \beta$ is enough large. Table?? shows expected signatures with the different NLSP type and its lifetime.

In the OPAL experiment, both the NLSP and the next-to-NLSP (NNLSP) signatures are considered. The NNLSP production events usually have less 
Table 1. The expected signatures for the different NLSP types and lifetimes. The symbols " $X$ " indicates additional particles from the cascade decays if sparticles heavier than the NLSP are produced.

\begin{tabular}{|l|c|l|}
\hline & neutralino NLSP & slepton NLSP \\
\hline \hline short lifetime & $\bullet$ acoplanar photons $+X$ & $\bullet$ acoplanar leptons $+X$ \\
\hline intermediate lifetime & $\bullet$ non-pointing photons $+X$ & $\begin{array}{l}\bullet \text { tracks with large impact parameters }+X \\
\bullet \text { kinked tracks }+X\end{array}$ \\
\hline long lifetime & $\bullet$ & $\bullet$ tracks with anomalous $d E / d x+X$ \\
\hline
\end{tabular}

Table 2. The topologies studied in the OPAL experiment are shown with the production and decay process for each NLSP.

\begin{tabular}{|l|l|l|}
\hline \multirow{2}{*}{ production } & \multicolumn{2}{|c|}{ decay } \\
\cline { 2 - 3 } & \multicolumn{1}{|c|}{ neutralino NLSP } & slepton NLSP \\
\hline$\tilde{\chi}_{1}^{0} \tilde{\chi}_{1}^{0}$ & $\gamma \gamma \tilde{G} \tilde{G}$ & $\tilde{\chi}_{1}^{0} \tilde{\chi}_{1}^{0} \ell^{+} \ell^{-} \rightarrow \ell^{+} \ell^{-} \ell^{+} \ell^{-} \tilde{G} \tilde{G}$ \\
\hline$\tilde{\ell}^{+} \tilde{\ell}^{-}$ & $\tilde{\chi}_{1}^{0} \tilde{\chi}_{1}^{0} \ell^{+} \ell^{-} \rightarrow \gamma \gamma \tilde{G} \tilde{G}+\ell^{+} \ell^{-}$ & $\begin{array}{l}\ell^{+} \ell^{-} \tilde{G} \tilde{G} \\
\ell^{+} \ell^{-}+\tilde{\chi}_{1}^{0} \tilde{\chi}_{1}^{0} \rightarrow \ell^{+} \ell^{-}+\tilde{\tau}^{+} \tau^{-} \tilde{\tau}^{-} \tau^{-} \\
\rightarrow \ell^{+} \ell^{-} \tau^{+} \tau^{-} \tau^{+} \tau^{-} \tilde{G} \tilde{G}\end{array}$ \\
\hline$\tilde{\chi}_{1}^{+} \tilde{\chi}_{1}^{-}$ & $\tilde{\chi}_{1}^{0} \tilde{\chi}_{1}^{0} \mathrm{~W}^{+} \mathrm{W}^{-} \rightarrow \gamma \gamma \tilde{G} \tilde{G}+\mathrm{f} \bar{f} f \bar{f}$ & \\
\hline$\tilde{\chi}_{2}^{0} \tilde{\chi}_{1}^{0}$ & $\tilde{\chi}_{1}^{0} \tilde{\chi}_{1}^{0} \mathrm{Z}^{0} \rightarrow \gamma \gamma \tilde{G} \tilde{G}+\mathrm{f} \overline{\mathrm{f}}$ & \\
\hline
\end{tabular}

background than those of the NLSP, and especially higher discovery potential in case of the NNLSP masses being close to the NLSP.

\subsection{NLSP productions}

$$
\begin{aligned}
& \tilde{\chi}_{1}^{0} \tilde{\chi}_{1}^{0} \rightarrow \gamma \gamma \tilde{G} \tilde{G} \\
& \tilde{\ell}_{1}^{+} \tilde{\ell}_{1}^{-} \rightarrow \ell^{+} \ell^{-} \tilde{G} \tilde{G}
\end{aligned}
$$

In case of the NLSP with short lifetime, main background are $\nu \nu \gamma(\gamma)$ and $\ell^{+} \nu \ell^{-} \nu$ for $\tilde{\chi}_{1}^{0} \tilde{\chi}_{1}^{0}$ and $\tilde{\ell}^{+} \tilde{\ell}^{-}$productions, respectively. The selection efficiency becomes high due to the "unbalanced" event shape as the NLSP mass is close to the beam energy (i.e. produced almost at rest). The $\tilde{\chi}_{1}^{0} \tilde{\chi}_{1}^{0}$ is produced dominantly via t-channel $\widetilde{e}_{i}^{ \pm}$exchange, which interferes with s-channel $\mathrm{Z}^{0}$ exchange. The $\mathrm{Z}^{0}$ exchange is possible only with higgsino components of $\tilde{\chi}_{1}^{0}$ 
which is usually small in the minimal case. The cross-section of $\widetilde{\mu}_{1}^{+} \widetilde{\mu}_{1}^{-}$production $\left(\widetilde{\tau}_{1}^{+} \widetilde{\tau}_{1}^{-}\right.$if $\tan \beta$ is small) is almost independent of model parameters, on the other hand, it depends on $\tilde{\chi}_{i}^{0}$ mass as well as their components in case of $\widetilde{e}_{1}^{+} \widetilde{e}_{1}^{-}$production. If the NLSP has a measurable lifetime, one can observe unique final states, which have tracks with a large impact parameter or kinked tracks. A faked signal arises from the SM particles with intermediate lifetime $\left(\mathrm{K}^{ \pm} \rightarrow \mu^{ \pm} \nu, \pi^{-} \pi^{0}\right.$ and $\left.\pi^{-} \rightarrow \mu^{ \pm} \nu\right)$ and the hadronic interaction with the detector material. Hadronic interactions and two-photon backgrounds are not well simulated, and are reduced by applying tight selections. In case of the slepton decaying out of the detector, tracks with anomalous ionization energy loss $d E / d x$ can be observed. There is nearly zero background since the variable, $d E / d x$, is very powerful to separate the signal from the SM background. The selection efficiency is kept around $80 \%$. Only one event with anomalous $d E / d x$ was observed with data of the luminosity $\sim 680 \mathrm{pb}^{-1}$ taken at $\sqrt{s}=183-208 \mathrm{GeV}$. If neutralino is pair-produced and they decay outside of the detector, nothing can be observed. The NNLSP productions might be available in this case. For instance, the analysis of two leptons plus missing energy has a sensitivity for $\widetilde{\ell}^{+} \widetilde{\ell}^{-}$production with long lifetime neutralino NLSP.

\subsection{NNLSP productions : neutralino NLSP}

$$
\begin{aligned}
\tilde{\chi}_{2}^{0} \tilde{\chi}_{1}^{0} & \rightarrow \tilde{\chi}_{1}^{0} \tilde{\chi}_{1}^{0} \mathrm{Z}^{0} \rightarrow \gamma \gamma \tilde{G} \tilde{G}+\mathrm{f} \overline{\mathrm{f}} \\
\tilde{\chi}_{1}^{+} \tilde{\chi}_{1}^{-} & \rightarrow \tilde{\chi}_{1}^{0} \tilde{\chi}_{1}^{0} \mathrm{~W}^{+} \mathrm{W}^{-} \rightarrow \gamma \gamma \tilde{G} \tilde{G}+\mathrm{f} \bar{f} \bar{f} \\
\tilde{\ell}_{1}^{+} \tilde{\ell}_{1}^{-} & \rightarrow \tilde{\chi}_{1}^{0} \tilde{\chi}_{1}^{0} \ell^{+} \ell^{-} \rightarrow \gamma \gamma \tilde{G} \tilde{G}+\ell^{+} \ell^{-}
\end{aligned}
$$

When the above processes occur, two photons plus something $(X)$ with missing energy can be observed. Requiring the two energetic isolated photons is very effective for distinguishing the signal from the background. Dominant faked signal photons are initial and final state radiative photons, and Bremsstrahlung photons. As the mass difference between the NNLSP and NLSP, $\Delta M$, is large, the sensitivity becomes low due to small photon energy and missing energy. In the minimal GMSB, $\tilde{\chi}_{2}^{0}$ and $\tilde{\chi}_{1}^{ \pm}$form a degenerate $W$-ino weak triplet and their mass differences from $\tilde{\chi}_{1}^{0}$ are around $20-50 \mathrm{GeV}$ within NNLSP production limits at LEP. On the other hand, wide mass difference is possible for slepton NNLSP. Several analyses optimized to different $X$ s and $\Delta M$ are adopted. The analyses do not restrict to the minimal case, the mass difference of from $3 \mathrm{GeV}$ up to the almost NNLSP mass are considered. The decay of $\tilde{\chi}_{2}^{0} \rightarrow \tilde{G} \gamma$ is apt to occur than $\tilde{\chi}_{2}^{0} \rightarrow \tilde{\chi}_{1}^{0} \mathrm{Z}^{0}$ with small 
$\Delta M$. But the signature is almost same to $\tilde{\chi}_{1}^{0} \tilde{\chi}_{1}^{0}$ with $\tilde{\chi}_{1}^{0}$ decaying into $\tilde{G} \gamma$. If selectron should be very heavy and the resulting cross-section of $\tilde{\chi}_{1}^{0} \tilde{\chi}_{1}^{0}$ is suppressed, some of these NNLSP productions might be effective.

\section{$2.3 \quad$ NNLSP productions : slepton NLSP}

$$
\begin{aligned}
& \tilde{\chi}_{1}^{0} \tilde{\chi}_{1}^{0} \rightarrow \tilde{\ell}_{1}^{+} \tilde{\ell}_{1}^{-} \ell^{+} \ell^{-} \rightarrow \ell^{+} \ell^{-} \ell^{+} \ell^{-} \tilde{G} \tilde{G} \\
& \tilde{\ell}_{1}^{+} \tilde{\ell}_{1}^{-} \rightarrow \ell^{+} \ell^{-} \tilde{\chi}_{1}^{0} \tilde{\chi}_{1}^{0} \rightarrow \ell^{+} \ell^{-} \tau^{+} \tau^{-} \tau^{+} \tau^{-} \tilde{G} \tilde{G}
\end{aligned}
$$

If the neutralino and sleptons (stau) are degenerate in their masses, the above processes play an important role. The NNLSP particle predominantly decays into the NLSP and its SM partner rather than decay into gravitino directly if it is kinematically allowed.

The 4-lepton analysis has a good sensitivity if the mass difference between neutralino and sleptons is roughly greater than $1 \mathrm{GeV}^{\text {? }}$. The 6-lepton final state is possible in case of the stau sole-NLSP (i.e. $\widetilde{\tau}_{1}^{ \pm}>\widetilde{e}_{1}^{ \pm} \sim \widetilde{\mu}_{1}^{ \pm}>m_{\tilde{\chi}_{1}^{0}}$ ). For the intermediated lifetime, the efficiency usually becomes lower than that of the short lifetime NLSP because the selection is tighten to reduce much two-photon and non-SM background.

\section{Summary}

The gauge-mediated SUSY Breaking model is attractive at both theoretical and experimental points. We have studied most of possible topologies and kinematics in GMSB models using high energy data, although no significant excess has been observed. All analyses and interpretation method has been already established. The all final results and the interpretation within the minimal GMSB model will be published.

\section{Acknowledgments}

I'd like to thank to Dr. R. Keeler and Dr. R. Mcphersn for their useful comments and advice.

\section{References}

1. E.Gildener, Phys. Rev. D 14 (1976) 1667;

E.Gildener and S.Weinberg Phys. Rev. D 14 (1976) 3333. 
2. M.Dine, W.Fischler and M.Srednicki, Nucl. Phys. B189 (1981) 575;

S.Dimopoulos and S.Raby, Nucl. Phys. B192 (1981) 353.

3. G.F.Giudice and R.Rattazzi, Phys. Rep. 322 (1999) 419

4. ALEPH Collaboration A. Heister et al. hep-ex/0203024, Submitted to Eur. Phys. J. C;

DELPHI Collaboration P. Abreu et al. Phys. Lett. B503 (2001) 34-48;

OPAL Collaboration B. Abbiendi et al. Phys. Lett. B501 (2001) 12

5. CDF Collaboration T. Affolder et al. Phys. Rev. Lett. 85 (2000) 1378;

D0 Collaboration B. Abbott et al. Phys. Rev. Lett. 80 (1998) 442.

6. OPAL Collaboration B. Abbiendi et al. Phys. Lett. B501 (2001) 12

7. OPAL Collaboration B. Abbiendi et al. Eur. Phys. J C14 (2000) 51

8. OPAL Collaboration B. Abbiendi et al. Eur.Phys.J.C18 (2000) 253

9. OPAL Collaboration, K. Ackerstaff et al. Phys. Lett. B433 (1998) 195

10. S.Ambrosanio, G.D.Kribs and S.P.Martin Nucl. Phys. B516 (1998) 55 